\title{
CMB Comptonization in Clusters: Spectral and Angular Power from Evolving Polytropic Gas
}

\author{
Sharon Sadeh ${ }^{1}$ \\ School of Physics and Astronomy, Tel Aviv University, Tel Aviv, 69978, Israel \\ and \\ Yoel Rephaeli \\ School of Physics and Astronomy, Tel Aviv University, Tel Aviv, 69978, Israel, \\ and \\ Center for Astrophysics and Space Sciences, University of California, San Diego, \\ La Jolla, CA 92093-0424
}

\begin{abstract}
The angular power spectrum of the Sunyaev-Zeldovich (SZ) effect is calculated in the $\Lambda$ CDM cosmological model with the aim of investigating its detailed dependence on the cluster population, gas morphology, and gas evolution. We calculate the power spectrum for three different mass functions, compute it within the framework of isothermal and polytropic gas distributions, and explore the effect of gas evolution on the magnitude and shape of the power spectrum. We show that it is indeed possible to explain the 'excess' power measured by the CBI experiment on small angular scales as originating from the SZ effect without (arbitrary) rescaling the value of $\sigma_{8}$, the mass variance parameter. The need for a self-consistent choice of the basic parameters characterizing the cluster population is emphasized. In particular, we stress the need for a consistent choice of the value of $\sigma_{8}$ extracted from fitting theoretical models for the mass function to the observed cluster X-ray temperature function, such that it agrees with the mass-temperature relation used to evaluate the cluster Comptonization parameter. Our treatment includes the explicit spectral dependence of the thermal component of the effect, which we calculate at various frequencies. We find appreciable differences between the nonrelativistic and relativistic predictions for the power spectrum even for this superposed contribution from clusters at the full range of gas temperatures.
\end{abstract}

Key words: Cosmology, CMB, Clusters of Galaxies

PACS: $98.65 . \mathrm{Cw}, 98.70 . \mathrm{Vc}, 98.65 . \mathrm{Hb}$

Preprint submitted to Elsevier Science

11 December 2017 


\section{Introduction}

The Sunyaev-Zeldovich (SZ) effect - the change in the cosmic microwave background $(\mathrm{CMB})$ intensity that results from Compton scattering in a cluster of galaxies - is a unique cosmological probe which is essentially independent of the cluster redshift. The effect has already been imaged in some $\sim 60$ clusters, yielding important information on such quantities as the gas mass fraction in clusters, and the Hubble constant, $H_{0}$. Multi-frequency measurements of the effect in many more clusters are expected in the near future when new SZ projects - both ground-based and stratospheric - will be operational. In addition to the detection of the effect in a large number of clusters, planned sky surveys, particularly by the Planck satellite, will likely map the CMB anisotropy induced by the effect. The much larger SZ database and the measurement of this anisotropy will greatly expand the scope of the effect as a cosmological probe due to its strong dependence on the cosmological and large scale parameters, and the morphology and evolution of clusters.

Extensive work in the last two decades (since it was first modeled [Rephaeli 1981] in the context of a simple model for IC gas evolution) has established the main features of SZ anisotropy in various cosmological, dark matter (DM), and cluster evolution models. For example, the SZ power spectrum was calculated in flat cold dark matter (e.g., SCDM, Atrio-Barandela \& Mücket 1999), MCDM (e.g., Komatsu \& Kitayama 1999), and open low-density (OCDM, Molnar \& Birkinshaw 2000) models (for reviews, see Rephaeli 1995a, Birkinshaw 1999, and Carlstrom et al. 2002). However, results for the power spectrum of this anisotropy and cluster number counts differ considerably even when calculated for the same cosmological and DM models, mostly due to differences in the modeling of the cluster mass function and gas properties, but in some cases also due to inconsistent choice of some of the many free parameters. There is a need to clarify some of these differences as part of the basic goal of a quantitative and realistic treatment of the SZ-induced anisotropy. This is clearly desirable also in order to optimize strategies for the spectral and spatial mapping of the anisotropy in the many surveys planned by ground-based and satellite experiments.

The measured values of CMB intensity differences across the sky naturally consist of contributions from all sources of anisotropy - primary, secondary, and tertiary. Most investigated is, of course, the primary anisotropy, originating in the very early universe, for which the SZ contribution could be regarded as a foreground 'contamination'. The latter, however, is very important for better understanding of the post-recombination universe, and in particular - to the global distribution of galaxy clusters and their physi-

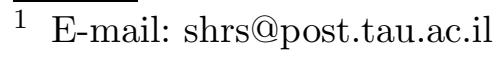


cal properties. The primary anisotropy has to be precisely known before the SZ (and other contributions) can be determined on angular scales of a few arcminutes. This is particularly important now that an 'excess' power was measured by the CBI experiment (Mason et al. 2002), at an estimated 3.1 $\sigma$ level, over what is predicted in standard models for the primary anisotropy at multipoles $\ell=2000-3500$. This 'excess' can possibly be due to the integrated SZ effect (Bond et al. 2002). The power spectrum was recently determined also by measurements with ACBAR (Goldstein et al. 2002) at $~ 150 \mathrm{GHz}$. Measurements at this and higher frequencies - well beyond the CBI 26-36 $\mathrm{GHz}$ band - are essential in order to exploit the unique spectral signature of the SZ effect as a powerful diagnostic tool by which it can be separated out from the primary anisotropy (e.g., Rephaeli 1995a, Cooray et al. 2000).

The calculation of the SZ power spectrum necessitates detailed modeling of the cluster mass function, intracluster (IC) gas properties and evolution. Consequently, the values of many parameters have to be specified in order to fully characterize clusters and their gaseous contents. Some of these parameters may be obtained from theoretical considerations (e.g. the critical overdensity for spherical collapse), while others are extracted from observations (e.g., cluster X-ray temperatures), or by comparing theoretical models with observational data, yielding important normalization factors, such as $\sigma_{8}$. This important parameter is the rms density field smoothed over a scale $8 h^{-1} \mathrm{Mpc}$, where $h$ is the Hubble constant in units of $100 \mathrm{~km} /(\mathrm{s} \mathrm{Mpc})$. In particular, the cluster mass-temperature relation and $\sigma_{8}$, which is usually estimated by means of the cluster X-ray temperature function, are coupled quantities which have to be consistently selected.

Since the angular power spectrum of the SZ effect has already been calculated in numerous papers, we focus here on various aspects of the calculation which have not yet been sufficiently explored. These include explicit form for the temporal of IC gas, and a more general polytropic spatial distribution of its temperature. The description of the cluster population is also extended to include two mass functions incorporating non-spherical collapse, in addition to the Press \& Schechter mass function. We also discuss the need for a consistent choice of normalization of the cluster density and mass-temperature relation. In our claculation we include the full spectral dependence of the thermal effect which we calculate at five frequencies (using the exact relativistic treatment) below, near, and above the crossover frequency (where the thermal component vanishes). These calculations underline the importance of using the exact relativistic expression for the change of intensity due to the SZ effect (Rephaeli 1995b), especially so near the crossover frequency.

Our basic computational approach is essentially similar to that presented in a few other papers (e.g. , Colafrancesco et al. 1997, Molnar \& Birkinshaw 2000); thus, our discussion of most aspects of the methodology will be brief. Rather, 
we will concentrate on issues that have attracted little or no attention. The paper is arranged as follows: in $\S 2$ we lay out the computational method, specifying the different parameters and scalings required in the calculation of SZ anisotropy. Results for the angular power spectrum are presented in $\S 3$, and discussed in $\S 4$.

\section{Methodology}

The calculation of CMB anisotropy induced by the SZ effect requires modeling of IC gas, the cluster population, and their evolution, as well as the parameters of the background cosmological model. We begin with a brief description of the effect itself: The thermal component of the effect is described by the change of the spectral intensity of the CMB across the cluster, $\Delta I_{t}$. In the nonrelativistic approximation, $\Delta I_{t}=i_{o} y g_{0}(x)$, where $i_{o}=2(k T)^{3} /(h c)^{2}, T$ is the CMB temperature, $y=\int\left(k T_{e} / m c^{2}\right) n \sigma_{T} d l$ is the Comptonization parameter, and $g_{0}(x)$ is the well known (Sunyaev \& Zeldovich 1972) spectral function. Here $n$ and $T_{e}$ are the electron number density and temperature, $\sigma_{T}$ is the Thomson scattering cross section, $d \ell$ is a line element along a line of sight (los) to the cluster, and $x=h \nu / k T$ is the non-dimensional frequency. This nonrelativistic expression for $\Delta I_{t}$ is not sufficiently accurate at high frequencies and at gas temperatures higher than a few $\mathrm{keV}$; a more exact relativistic description is then required (Rephaeli 1995b). As shown in the latter paper, the more exact relativistic calculation $\Delta I_{t}$ differs appreciably from the non-relativistic expression, particularly so near the crossover frequency, $x_{0}$. This has practical ramifications since $x_{0}$ is the optimal frequency for measurement of the cluster velocity (in the CMB frame) along the los, and the fact that this frequency is extremely suitable for CMB observations, as it allows the subtraction of (the dominant) thermal component from the full CMB signal.

\subsection{IC Gas Properties}

In all calculations it is assumed that IC gas is in a state of hydrostatic equilibrium in the gravitational potential of the cluster. The density profile adopted here is the commonly used isothermal $\beta$ model

$$
n(r)=n_{0}\left[1+\left(\frac{r}{r_{c}}\right)^{2}\right]^{-3 \beta / 2},
$$

where $r_{c}$ is the core radius. For simplicity we take the cluster radial extent roughly, the virialization radius - to be $p r_{c}=10 r_{c}$. The core radius is scaled such that $r_{c}=0.15 h^{-1} M p c$ for a local cluster with mass $10^{15} h^{-1} M_{\odot}$. 
The gas mass is assumed to constitute a fraction $f$ of the total cluster mass. For local clusters of mass $10^{15} M_{\odot} h^{-1}$, we will scale to the nominal value $f=0.1$, in accord with recent SZ results (Carlstrom et al. 2002). The fact that the gas is appreciably metal-enriched, with typical iron abundance of $\sim 0.2-0.3$ solar, is indicative of galactic and intergalactic origins and varying relative contributions. Temporal evolution of the gas must then be taken into account; we do so by the parameterization $f(M, z) \propto M^{\eta} t^{\xi}(z)$, with $t$ denoting the age of the universe at redshift $z$. Some evidence for the form of this evolution was seen in cluster measurements made during the Einstein Medium Sensitivity Survey (EMSS; Gioia et al. 1990, Henry et al. 1992). Analysis of the EMSS results led Colafrancesco \& Vittorio (1994) to the deduction of the approximate values $\xi=1.45$, and $\eta=0.2$, which we adopt here. Clearly, an increase of IC gas fraction with mass, and a decrease with $z$ is indicated by these values.

We consider general polytropic models $T_{e} \propto n^{\gamma-1}$ which include the special case of isothermal distribution $(\gamma=1)$. The integrated $y$ parameter along a los forming an angle $\theta$ with a los pointing at the cluster center is given by

$$
\begin{array}{r}
y(\theta)=\frac{y_{0}}{r_{c}(M, z)} \int_{0}^{r_{1}} \frac{d \ell}{\left(1+\ell^{2}+\left(\frac{\theta}{\theta_{c}}\right)^{2}\right)^{\delta}} \\
=y_{0}\left[1+\left(\frac{\theta}{\theta_{c}}\right)^{2}\right]^{-\delta} r_{12} F_{1}\left[\frac{1}{2}, \delta ; \frac{3}{2} ;-\frac{r_{1}^{2}}{1+\left(\theta / \theta_{c}\right)^{2}}\right] .
\end{array}
$$

Here $y_{0}=\frac{2 k_{B} \sigma_{T}}{m_{e} c^{2}} n_{0}(M, z) r_{c}(M, z) T_{0}(M, z), r_{1}=\sqrt{p^{2}-\left(\theta / \theta_{c}\right)^{2}}, \delta=3 \beta \gamma / 2$, and ${ }_{2} F_{1}$ is the Hypergeometric function. In the simpler isothermal case we adopt $\beta=2 / 3$, and obtain

$$
y(\theta)=\frac{y_{0}}{\sqrt{\left(1+\left(\theta / \theta_{c}\right)^{2}\right.}} \tan ^{-1} \sqrt{\frac{p^{2}-\left(\theta / \theta_{c}\right)^{2}}{1+\left(\theta / \theta_{c}\right)^{2}}} .
$$

\subsection{Cluster SZ Profile}

The calculation of the SZ angular power spectrum involves a number of stages. Here we only outline the method; further details can be found in previous works (e.g., Colafrancesco et al. 1997, Molnar \& Birkinshaw 2000). The relative temperature change along a los to the cluster is

$$
\frac{\Delta T}{T}=\left[x \operatorname{coth}\left(\frac{x}{2}\right)-4\right] \cdot y(\theta)=s(x) \cdot y(\theta)
$$


where $x=h \nu / k T$ is the non-dimensional frequency, and $y(\theta)$ is the Comptonization parameter along a los displaced an angle $\theta$ from the center of the cluster. For isothermal gas with a King profile we have

$$
\begin{aligned}
& y(\theta)=2 \frac{k \sigma_{T}}{m_{e} c^{2}} n(0)(z, M) T(0)(z, M) r_{c}(z, M) \frac{1}{\sqrt{1+\left(\theta / \theta_{c}\right)^{2}}} \\
& \cdot \tan ^{-1}\left[p \sqrt{\frac{1-\left(\theta / p \theta_{c}\right)^{2}}{1+\left(\theta / \theta_{c}\right)^{2}}}\right],
\end{aligned}
$$

where $\theta_{c}$ denotes the angular diameter subtended by the cluster core. Expressions for the temperature, density and cluster core radius are given in Colafrancesco et al. (1994) for flat cosmological models, equations (6), (9) and (10), and the equivalent relations in equations (6) and (7) in Colafrancesco et al. (1997) for low-density models. For polytropic gas the corresponding expression is

$$
y(\theta)=y_{0}\left[1+\left(\frac{\theta}{\theta_{c}}\right)^{2}\right]^{-\delta} r_{12} F_{1}\left[\frac{1}{2}, \delta ; \frac{3}{2} ;-\frac{r_{1}^{2}}{1+\left(\theta / \theta_{c}\right)^{2}}\right] .
$$

The spectral dependence of (the thermal component of) $\Delta T$, which is given by the function $s(x)=x \operatorname{coth}(x / 2)-4$, is only valid at low cluster temperatures. As noted, a relativistically correct expression has to be used for most of the cluster temperature range and at high frequencies. Approximate analytic expressions for the relativistic case have been obtained, applicable at temperatures $k_{B} T \leq 15 \mathrm{keV}$ (e.g., Itoh et al. 1998, Shimon \& Rephaeli 2003). The computation of the SZ angular power spectrum involves an integration over the mass-redshift distribution of the cluster population, and thus clusters in a wide range of temperatures - determined by the masstemperature (and redshift) scaling - contribute to the power spectrum. The predicted range of gas temperatures can be estimated by using the usual scal$\operatorname{ing} T=7.76 \beta^{-1}\left(\frac{M}{10^{15} h^{-1} M_{\odot}}\right)^{2 / 3}(1+z) k e V$, in a flat $\Omega_{0}=1 \mathrm{CDM}$ model (Molnar \& Birkinshaw 2000). Since $\beta$ assumes values in the range $\sim 0.5-1$, and $M$ can be as high as $10^{16} h^{-1} M_{\odot}$, it follows that this relation yields high temperatures $(>30 \mathrm{keV})$ at $z>1$. While a naive use of this scaling relation at $z>1$ is likely to be improper - due (among other considerations) to fast radiative cooling in the high density cores of very massive clusters, if these existed at all already at such early times (contrary to expectations of hierarchical growth by merging of sub-clusters) - it is clear that we have to use the relativistically correct spectral distribution of the SZ effect. It is nonetheless obvious that the mass function yields relatively low number of clusters with high temperatures, and that their overall contribution to the power spectrum 
is likely to be small.

\subsection{Cluster Mass Functions}

We adopt the PS mass function (Press \& Schechter 1974) as the 'standard' model for characterizing the cluster population. However reasonable and successful this function is, it still suffers from a number of drawbacks. From a theoretical point of view, the function has been derived using the oversimplified assumption of spherical infall, which lies on weak theoretical grounds. Its failure to deal with secondary infall is yet another well-known shortcoming. It has been shown that other theoretically derived mass functions are more consistent with results of N-body simulations. Specifically, Lee \& Shandarin (1999), and Sheth \& Tormen (1999) (hereafter LS \& ST, respectively) have derived mass functions incorporating non-spherical collapse. We calculate the SZ angular power spectrum based on all these three mass functions and assess the differences between the respective values of $C_{\ell}$.

For the mass function to correctly quantify the cluster abundance in the massredshift space, the normalization of the power spectrum of the density fluctuation field must be determined. This is usually specified in terms of $\sigma_{8}$, the rms density field smoothed over a scale $8 h^{-1} \mathrm{Mpc}$, a free parameter in the analytic mass function expression whose best-fit value can be extracted from the observed X-ray temperature function. The temperature function describes the abundance of clusters $N(T)$ with temperature $T$ within a small interval $d T$; this is the differential form of the function. The integral form $N(>T)$ specifies the population of clusters with temperatures equal to or higher than $T$. Fitting a mass function to the temperature function requires a mass-temperature calibration, ascribing a temperature $T(M)$ to a cluster with mass $M$. The parameter $\sigma_{8}$ obviously depends on the adopted calibration. We first explain this dependence intuitively, then point at likely consequences of using a normalization which stems from an inconsistent calibration.

Consider two temperature-mass relations (TMR) denoted by $T_{1}(M)$ and $T_{2}(M)$, satisfying $T_{1}(M)>T_{2}(M)$ for all $M$. Since any TMR predicts higher temperatures with increasing mass, i.e., the temperature is a monotonically increasing function of mass, the inverse mass-temperature relations (MTR), $M_{1}(T)$ and $M_{2}(T)$, must satisfy $M_{1}(T)<M_{2}(T)$ for all $T$. Consider now an observed temperature function $N(T) d T$, which has to be related to the mass function $(\mathrm{MF})$. The latter is characterized by the normalization factor $\sigma_{8}$, and a fit of the presumed MF to the observed temperature function using a TMR may be performed with the normalization $\sigma_{8}$ as the fit parameter. The temperature 
function may be written in terms of the MF as follows:

$$
N(T) d T=N[M(T)] \frac{d T}{d M} d M
$$

and

$$
N[M(T)] d M=N(T) \frac{d M}{d T} d T=N(T) d T \frac{3 M}{2 T},
$$

where the last equality follows from the relation $T \propto M^{2 / 3}$. Given two MTRs the corresponding MF predictions are

$$
\left\{\begin{array}{l}
N\left[M_{1}(T)\right] d M=N(T) d T \frac{3 M_{1}(T)}{2 T} \\
N\left[M_{2}(T)\right] d M=N(T) d T \frac{3 M_{2}(T)}{2 T} .
\end{array}\right.
$$

However, to be able to compare their predictions, the arguments of these functions must be identical. Defining $M_{1}(T)=M_{2}\left(T^{*}\right)$ we obtain the following set of equations:

$$
\left\{\begin{array}{l}
n\left[M_{1}(T)\right] d M=n(T) d T \frac{3 M_{1}(T)}{2 T} \\
n\left[M_{2}\left(T^{*}\right)\right] d M=n\left(T^{*}\right) d T \frac{3 M_{2}\left(T^{*}\right)}{2 T^{*}} .
\end{array}\right.
$$

Now the arguments of the two MFs are identical but the MFs predictions themselves are different, and since $M_{1}(T)<M_{2}(T) \forall T$, it turns out that in order for the condition $M_{1}(T)=M_{2}\left(T^{*}\right)$ to be satisfied, one must have $T^{*}<T$. This means that the lower expression in equation set (10) predicts higher cluster abundances. We may thus conclude that a MF derived using a MTR which associates a lower mass with a given temperature would predict a higher cluster abundance at the corresponding mass.

The MF is to a good approximation a monotonically increasing function of $\sigma_{8}$, technically due to the fact that $\sigma_{8}$ appears in the denominator of the exponential part of $N(M)$. Only in the small mass range does the multiplicative term $1 / \sigma_{8}$ dominates its behavior of the MF; in this range the MF is a decreasing function of $\sigma_{8}\left[N(M) \propto \sigma_{8}^{-1} \exp \left(-\delta_{c}^{2} / 2 \sigma^{2} \sigma_{8}^{2} D_{z}^{2}\right)\right]$. Specifically, $N(M)$ is an increasing function of $\sigma_{8}$ for $\sigma_{M} \cdot D_{z}<\delta_{c}$, where $\sigma_{M}, D_{z}$ and $\delta_{c}$ are the (unnormalized) mass variance smoothed over mass scale $M$, the linear growth 
factor of density fluctuations, and the critical density for spherical collapse, respectively. We can now predict the consequences of employing a normalization factor $\sigma_{8}$ extracted using a specific MTR, but using a different MTR for specifying the temperature ascribed to a cluster of mass $M$, required for the evaluation of the cluster $y$-parameter $\propto T_{0}$. Note that the SZ angular power spectrum is proportional to the product of the MF evaluated at specific points in the mass-redshift space and the temperature (through the $y$-parameter dependence). Suppose that the normalization parameter $\sigma_{8}$ is extracted by fitting a theoretical MF to an observed temperature function using a TMR $T_{1}(M)$, and the gas temperature used in the evaluation of the cluster Comptonization parameter is derived from a TMR $T_{2}(M)$, such that $T_{1}(M)>T_{2}(M)$. As seen before, the $T_{1}(M)$ scaling predicts lower cluster abundances than would be predicted employing the $T_{2}(M)$ scaling, and the $T_{2}(M)$ scaling attributes lower temperatures to a given mass. This could lead to an appreciable underestimation of the SZ $C_{\ell}$ 's. In the opposite case, when $T_{2}(M)$ is used to extract $\sigma_{8}$, and $T_{1}(M)$ to evaluate the temperature, $C_{\ell}$ will likely be overestimated. This inconsistency underlines the need for a unique choice of the TMR.

\subsection{Angular Power Spectrum}

The angular power spectrum of the SZ effect (e.g., Molnar \& Birkinshaw 2000) in the cluster population is characterized by

$$
C_{\ell}=\int_{z} r^{2} \frac{d r}{d z} \int_{M} N(M, z) \zeta_{\ell}(M, z) d M d z,
$$

where $N(M, z)$ is the $\mathrm{MF}$, and $r$ is the comoving radial distance. The function $\zeta(M, z)$ is the angular Fourier transform of the spatial profile of $\Delta T$, which in the nonrelativistic limit is given in terms of the profile of the Comptonization parameter (eq. 5). In our calculations we consider clusters in the mass range of $10^{13}-10^{16} M_{\odot} h^{-1}$, but discuss how the results change when a narrower range is taken. The redshift integration interval - determined by the earliest epoch when clusters can be assumed to have formed and virialized - is uncertain and clearly depends on the cosmological model.

\section{Results and Discussion}

The 'standard' model in our calculations of the SZ angular power spectrum is the currently favored flat, vacuum-dominated universe. The model is characterized by the following set of cosmological parameters: $\Omega_{m}=0.3, \Omega_{\Lambda}=$ 
$0.7, \Omega_{B}=0.05, n=1, h=0.7$. Clusters are assumed to be distributed according to the PS MF, have isothermal gas over a spherical region with a radius of $10 r_{c}$, with the gas mass fraction that scales with the cluster mass and evolves in time as $f(M, z) \propto M^{0.2} t(z)^{1.45}$. We have also used two other recently proposed MFs, and generalized the gas distribution to a polytrope with a range of values for the index. In the calculations we have also considered the case of no gas evolution. The various cases of gas parameters for a given cluster are treated self-consistently by keeping the total mass and the gas mass fraction fixed as the polytropic index is varied. We carried out the calculations both without and with the SZ spectral dependence (the latter only for our standard model) evaluated at five observing frequencies - taken to correspond to the four MITO channels (De Petris et al. 2002) and the $545 \mathrm{GHz}$ channel of the HFI on the Planck satellite.

As mentioned in section 2.3, a mass-temperature scaling is needed in order to fit a MF to the observed cluster X-ray temperature function, so as to obtain the normalization $\sigma_{8}$. We have adopted the mass-temperature relation and the deduced values of $\sigma_{8}$ obtained by $\mathrm{Wu}$ (2000) from fits of the LS and ST MF to the observed temperature function derived by Viana \& Liddle (1999). The latter authors relaxed the assumption of spherical gravitational collapse, and derived analytic expressions within the framework of non-spherical collapse. (We note that in these models a halo may begin to form when an overdensity collapses along one of the principal axes of the deformation tensor. The earlier formation of clusters would delay the active formation epoch in comparison with the PS case [Wu 2000], i.e., a cluster with present virial mass $M$ has a higher probability of forming at a lower redshift than in the PS models.) For our choice of the standard model parameters, the values of $\sigma_{8}$ are $0.974,0.839,0.913$ for the PS, LS and ST MFs, respectively. This parameter was recently deduced also from the SDSS and WMAP CMB anisotropy databases, yielding somewhat lower values than we have adopted here (Melchiorri et al. 2002, Bennett et al. 2003); however, the range of uncertainties in the various deduced values of $\sigma_{8}$ is wide and certainly includes the above three values that we use here. Results for the power spectrum for the standard model with full, partial, and no gas evolution (to be specified below) are plotted in figure 1. Note that here and in the following figures the power spectrum is expressed in its dimensionless form, namely it is $\Delta T / T_{0}$ (rather than $\Delta T$ ) that is expanded in Fourier space. In Figure 2, power spectra are shown for two polytropic models with indices $\gamma=1.1,1.3$, and for the standard isothermal model. The predictions from the PS, LS and ST mass functions are shown in figure 3. The primary and SZ induced anisotropy power spectra are shown in figure 4 .

The SZ power spectrum in the standard model and in the models with the LS and ST MFs are shown in figure 3. Note that all three curves lie close to each other, particularly the PS and ST MFs. The LS MF yields lower values for 
the power spectrum; as can be seen in the right panel of figure 3, this amounts to a reduction of the power spctrum by $\sim 50 \%$, somewhat higher at low multipoles, and lower by $\sim 10 \%$ at low multipoles; this curve virtually coincides with the PS prediction at higher multipoles. Apparently, when these MFs are fit to the same observational data by using the same mass-temperature relation, differences among the power spectra will be minor. This is in particular important due to the fact that the MF is very sensitive to the input parameters, in particular to those appearing in its exponential part. A substantial amount of effort has been made over recent years to improve the PS model by resolving some of its problematic features, and to obtaining more realistic MFs. It appears that at least for the task of calculating SZ power spectra, using these functions instead of the PS MF does not affect much the results, particularly so when compared with other sources of uncertainties.

In all the models considered here the SZ power spectrum exhibits the same general behavior: a monotonic increase from low levels on large scales (low $\ell$ ), with a steep rise to a peak centered around $\ell \sim 1500-3000$ (depending on the model), followed by a steep decline. Differences between the respective curves are manifested in the orientation of the curve relative to the multipole axis and the magnitude - or height - reached at the peak. This characteristic curve stems from the angular Fourier transform of the Comptonization parameter. For small values of $\ell$ the Fourier transformed profile is essentially constant in magnitude; the parabolic shape is due to multiplication by $\ell(\ell+1)$. On smaller scales the Fourier transformed profile is exponentially damped, becoming sensitive to the internal structure of the cluster. This gives rise to the steep fall of the power spectrum; the peak of the curve roughly represents the transition between large and small scales, or between unresolved and resolved clusters. Note that the power spectrum is calculated only for the thermal component of the SZ effect; the related anisotropy induced by the kinematic component has been treated in various papers (e.g., Cooray 2001). The magnitude of the integrated contribution of this component is second order in the cluster velocity (in the CMB frame), and thus very much smaller than the respective contribution of the main, thermal component.

Gas evolution is a major factor determining the shape of the power spectrum, as is apparent from figure 1; with our adopted form of gas evolution the curve peaks at $\ell \sim 1400$, reaching a magnitude of $\sim 2.2 \cdot 10^{-11}$. Without gas evolution the peak shifts considerably to $\ell \sim 8000$, where it reaches the much higher value of $\sim 2.4 \cdot 10^{-10}$. Comparison of the two curves reveals that the higher the multipole, the greater is the contrast between the powers of the nonevolving and evolving gas. This stems from the higher gas densities in distant clusters predicted in the non-evolutionary gas scenario, which gives rise to an increase of power on smaller scales, reflecting the small angular sizes of distant clusters. It is also interesting to calculate the power spectrum in two 'partial' gas-evolving models, the first chosen to correspond to $\xi=0$ and 
$\eta=0.2$, and the second to $\xi=1.45$ and $\eta=0$ - namely removing from the gas mass fraction the redshift and mass dependences, respectively - in order to gauge the effect of the range of values of these parameters on the calculated power. For example, removing the redshift dependence results in a considerable dependence of the results on the mass lower limit, since - as can be seen in figure 1 - the non-evolving gas model still dominates the partially evolving gas model by a factor of $\sim 17$ at the highest multipoles. This maximal factor corresponds to the lowest cluster mass considered in the calculation, $M=10^{13} M_{\odot} h^{-1}$; this factor would have been smaller had we taken a higher mass cutoff.

The $\eta=0$ curve provides insight into the redshift range where most contributing to the anisotropy. Since the power spectrum in the model with non-evolving gas is only a factor of $\sim 2$ larger (on average) than that of evolving gas, we can evaluate the maximal redshift at which the contribution is still appreciable: In a flat universe we have

$$
\frac{t}{t_{0}}=\frac{\ln \left[\frac{\Omega_{\Lambda}^{1 / 2}+\left[\Omega_{\Lambda}+\Omega_{m}(1+z)^{3}\right]^{1 / 2}}{\Omega_{m}^{1 / 2}(1+z)^{3 / 2}}\right]}{\ln \left[\frac{\Omega_{\Lambda}^{1 / 2}+1}{\Omega_{m}^{1 / 2}}\right]} .
$$

Now, the ratio between the power spectra excluding gas evolution and including only its temporal dependence is, $\left(t / t_{0}\right)^{-\xi}$, and with the best fit value $\xi=1.45$, we determine the maximal factor this term contributes to the power spectrum in the non-evolving scenario to be $\left(t / t_{0}\right)^{-1.45} \sim \sqrt{2}$. Employing equation $(12)$ to solve for the redshift yields $z \approx 0.24$. The relatively low value of this redshift may seem surprising as we may expect high-redshift objects to contribute considerably to the power spectrum when there is no gas evolution. Instead, we only see a slight enhancement of its intensity, which peaks around a rather low value. One may then conclude that the ever decreasing cluster population at higher redshifts is responsible for this effect, and that the scaling of IC gas with mass dominates over its temporal change.

It is unrealistic to expect that thermal conductivity in the gas is sufficiently high for it to be isothermal over the full extent of the gas distribution. A more general distribution is that of polytropic gas a polytropic equation of state, $P \propto \rho^{\gamma}$, for which the Comptonization parameter $y$ scales as a los integral of $n^{\gamma}$. Clearly, for isothermal gas and $\beta=2 / 3, \delta=1$ (eq. 2). We consider two values of the polytropic index: $\gamma=1.1,1.3$, in addition to the isothermal case. Using the hydrostatic equation, the central temperature is

$$
T_{0}=\frac{G \mu m_{H}\left(1+p^{2}\right)^{\delta}}{k_{B} \rho_{0}\left[\left(1+p^{2}\right)^{\delta}-1\right]} \frac{\psi^{\prime}(p, \beta)}{\omega(p, \beta)}
$$


the functions $\psi^{\prime}(p, \beta)$ and $\omega(p, \beta)$ are defined in Colafrancesco et al. (1994). While the central temperature is not sensitive to the exact value of $\gamma$, the Comptonization parameter has a steeper distribution in the polytropic case, resulting in a substantially different power spectrum than that of isothermal gas. Figure 2 describes the SZ angular power spectrum in the isothermal and the two other polytropic cases. While there is a slight increase of the power for $\gamma=1.1$ (as compared to the isothermal case), the peak value for $\gamma=1.3$ is a factor $\sim 2$ lower with respect to the isothermal gas. The peaks shift to higher values of $\ell$ in both ploytropic cases. These changes result from a steeper Comptonization parameter due to a more compact cluster, leading to smaller cluster size and thus an increase of power on smaller scales. The peaks of the two curves for polytropic models with $\gamma>1$ occur in the range $\ell \approx 2000-3000$; the steeply falling part of the profiles shifts further to the right along the multipole axis. The difference between the magnitudes in the two polytropic cases is likely due to an interplay between two competing effects: the steeper Comptonization parameter assumes higher values in the central cluster regions, but it assumes these values along shorter distances. Therefore, with polytropic indices slightly above unity, there is an increase of magnitude, where the steeper profile dominates over the somewhat shorter distances along lines of sight with constant values of $y$, while for higher values of $\gamma$ the impact of the reduced pathlength dominates.

In figure 5 we plot the primary anisotropy, calculated using the CMBFAST code, and show the results from WMAP, CBI, and ACBAR measurements, as well as SZ power spectra in the isothermal and polytropic cases considered above. We have included the spectral dependence which we calculated at a frequency of $31 \mathrm{GHz}$, as appropriate for the CBI experiment. ACBAR measurements (Kuo et al. 2002) were at a frequency of $150 \mathrm{GHz}$; the data points are shown as reported (in the latter paper). Were it known that the signal measured at high multipoles is largely due to the SZ effect, we could have easily scaled the ACBAR data to the lower CBI frequency by the theoretically predicted spectral factor of $\sim 4.2$ (assuming a mean gas temperature of 7 $\mathrm{keV}$ ). This would have resulted in an enhancement of the ACBAR measured power over the data collected at the actual observing frequency.

The 'excess' power detected by the CBI experiment at high multipoles (Mason et al. 2002) may possibly be attributed to SZ signal. It has been suggested that this measured 'excess' is likely to require a modification of $\sigma_{8}$, in order to increase the cluster population, as predicted by the PS mass function. However, such a modification is not that simple to make; $\sigma_{8}$ is usually estimated by fitting a theoretical mass function to the observed temperature function of $\mathrm{X}$-ray clusters; therefore, its value is set by the fit. Moreover, different analyses yielded various values which span the rather broad range, $0.7 \lesssim \sigma_{8} \lesssim 1$. An arbitrary modification of this parameter would have consequences on the mass-temperature relation, which might be in total disccordance with the 
original mass-temperature relation employed in order to extract $\sigma_{8}$ in the first place. Our results seem to be consistent with the CBI measurements, thus circumventing the need for a modification in this parameter. However, even if this was not the case, discrepancies between the two sets of results may be explained by other means than the normalization parameter $\sigma_{8}$. In particular, the not fully known properties of IC gas provide a certain degree of freedom in adjusting the theoretically predicted level of the anisotropy. For exmaple, as we have shown above, the anisotropy depends apprecaibly on the evolution of IC gas; moderate (or no) evolution results in a considerable increase of the power spectrum at high multipoles. This is particularly relevant given the observational indications that there is weak or no evolution of the X-ray luminosity function of clusters.

In figure 4 we compare the SZ power spectra obtained in the $\Lambda$ CDM model with the corresponding power spectra of the primary anisotropy. The latter was computed using the CMBFAST code (Zaldarriaga \& Seljak 1996). As can be seen from the figure, the SZ power spectrum begins to dominate over the primary anisotropy at multipoles of $\ell \sim 2000$, but even at lower multipoles $(\ell \gtrsim 1500)$ the magnitude of the SZ power already constitutes a fraction $(\gtrsim 10 \%)$ of the primary power. Furthermore, these results were obtained with constant spectral function, $s(x)=1$. Its value in the Rayleigh-Jeans region is -2 , but it can be much larger; for example at the highest frequency of the HFI instrument of Planck ( $\sim 857 \mathrm{GHz}), s(x) \simeq 11$, so - from merely a theoretical point of view the - there is a clear advantage in measuring the power at high frequencies.

The power spectrum was calculated at five frequencies, four of which correspond to the channels, $x=2.5,3.9,4.8,6.2$, and a high frequency - the highest HFI channel with reasonably adequate sensitivity to SZ measurements aboard the PLANCK satellite, $\nu=545 G H z, x=9.6$. To assess the degree of importance of using the relativistic SZ expression we plot the SZ angular power spectrum obtained using the non-relativistic expression, the exact relativistic expression, and the approximate analytic expression of Itoh et al. (1998): figure 6 compares the SZ angular power spectrum calculated with the nonrelativistic expression of the spectral distortion with that calculated using the exact relativistic expression. The results demonstrate (again) the need for using the exact relativistic expression. The approximate analytic expression is of limited use near the the crossover frequency, as shown in figure 7 . For example, at $x=2.511$ and $x=3.907$ and high values of $\ell$, it yields values that are $25 \%-200 \%$ larger than those calculated using the exact relativistic treatment.

Finally, to determine the redshift distribution of the SZ power spectrum contribution of clusters, we divide the redshift integration range $(0.01 \leq z \leq 6)$ into six intervals, and plot in figure 8 the fractional contribution of each in- 
terval to the overall power spectrum at $x=2.5$ (the corresponding plots at the other frequencies do not differ substantially). As can be seen in the figure, the contribution to the power spectrum from redshifts $z>1$ is negligible at multipoles $\ell \lesssim 1000$. The contribution of clusters at $1<z<2$ increases from $\sim 10 \%$ at $\ell \sim 2000$, to as much as $40 \%$ at the highest multipoles. Clusters at $2<z<3$ contribute only at the highest multipoles at a level $\leq 7 \%$, and the contribution of clusters at still higher redshifts is $<1 \%$, which is the reason for its absence in the figure. These results agree with the analysis done based on the partial gas-evolution scenario discussed earlier in this section. These results also imply that even though high gas temperatures are predicted at $z>1$ by the virial mass-temperature relation, which is based on a naive redshift scaling, the impact of this unrealistic relation on our results is minimal since the contribution of high temperature clusters is negligible.

\section{Conclusion}

One of the main goals of our work has been to understand the reasons for the substantial variance among previous predictions for the power spectrum of the SZ-induced anisotropy. Among the various works on the power spectrum which employ analytic forms of the mass function to calculate the power spectrum in the context of a given cosmological and DM model, the main reason for quantitative differences was shown to be a choice of the cluster masstemperature relation that is inconsistent with value of $\sigma_{8}$ which is adopted in the calculation. We have taken explicit account of the evolution of the gas fraction in the context of a simple model, and explored how the power spectrum depends on the index of a polytropic equation of state. Our results are therefore self-consistent, more general in the descriptionof the properties of the gas than previous treatments, and include detailed predictions of the power spectrum at five frequencies based on a relativistic calculation of the spectral distribution of the SZ effect. 


\section{References}

Atrio-Barandela F. \& Mücket J.P. 1999, ApJ. , 515, 465. (1999ApJ . . 515 . .465A)

Bennett et al., astro-ph/0302207

Birkinshaw M. 1999, Phys.Rep. , 310, 97

Bond et al., astro-ph/0205386

Carlstrom J.E., Holder G.P., \& Reese E.D. 2002, Annu. Rev. Astron. Astrophys. , 40, 643. (2002ARA\&A . .40 . 643C)

Colafrancesco S. \& Vittorio N. 1994, ApJ. , 422, 443. (1994ApJ . . 422 . .443C)

Colafrancesco S., Mazzotta P., Rephaeli Y., \& Vittorio N. 1994, ApJ. , 433, 454. (1994ApJ . . 433. .454C)

Colafrancesco S., Mazzotta P., Rephaeli Y., \& Vittorio N. 1997, ApJ. , 479, 1. (1997ApJ . . 479 ....1C)

Cooray A. 2001, Phys. Rev. D 063514. (2001PhRvD. .64f3514C)

Cooray A., Hu W., \& Tegmark M. 2000, ApJ. , 540, 1. ApJ. , 356, 32. (2000ApJ ...540 ....1C)

De Petris et al., 2002, ApJ. Lett. , 574, L119. (2002ApJ . .574L.119D)

Gioia I.M. et al., 1990, ApJ. Lett. , 356, L35. (1990ApJ . . 356L. . 35G)

Goldstein et al., astro-ph/0212517

Henry J.P. et al., 1992, ApJ. , 386, 408. (1992ApJ . . .386 . .408H)

Itoh N., Kohyama Y., \& Nozawa S. 1998, ApJ. , 502, 7. (1998ApJ . . 502 . . . 7I)

Komatsu E. \& Kitayama T. 1999, ApJ. Lett. , 526, L1. (1999ApJ . . 526L. . . 1K)

Lee J. \& Shandarin S.F. 1998, ApJ. , 500, 14. (1998ApJ . . 500 . . 14L)

Mason et al., 2003, ApJ. , 591, 540. (2003ApJ. . .591 . .540M)

Melchiorri, A., Bode, P., Bahcall, N.A., Silk, J., 2003, ApJ. , 586, 1. (2003ApJ . . 586L . . . 1M)

Molnar S.M. \& Birkinshaw M. 2000, ApJ. , 537, 542. (CambridgeUniversity Press) (2000ApJ . . 537 . 542M)

Peebles P.J.E. 1993, Principles of Physical Cosmology, (Princeton University Press)

Press W.H. \& Schechter P. 1974, ApJ. , 187, 425. (1974ApJ . . 187. .425P)

Rephaeli Y. 1981, ApJ. , 245, 351. (1981ApJ. . . 245 . .351R)

Rephaeli Y. 1995a, Annu. Rev. Astron. Astrophys. 33, 541. (1995ARA\&A. . 33. . 541R) 
Rephaeli Y. 1995b, ApJ. 445, 33. (1995ApJ . . 445 . . 33R)

Sheth R.K., Tormen G., 1999, MNRAS , 308, 119. (1999MNRAS.308..119S)

Shimon M. \& Rephaeli Y., 2003, astro-ph/0309098

Sunyaev R.A. \& Y.B. Zeldovich 1972, Comments Astrophys.Space Phys. 4, 173. (1972ComAp...4.173S)

Viana P.T.P. \& Liddle A.R., 1996, MNRAS , 281, 323. (1996MNRAS.281 . 323V)

Wu J-H. P., 2001, MNRAS , 327, 629. (2001MNRAS.327 . 629W)

Zaldarriaga M. \& Seljak U., 1996, http://www.sns.ias.edu/matiasz/CMBFAST/cmbfast.html 

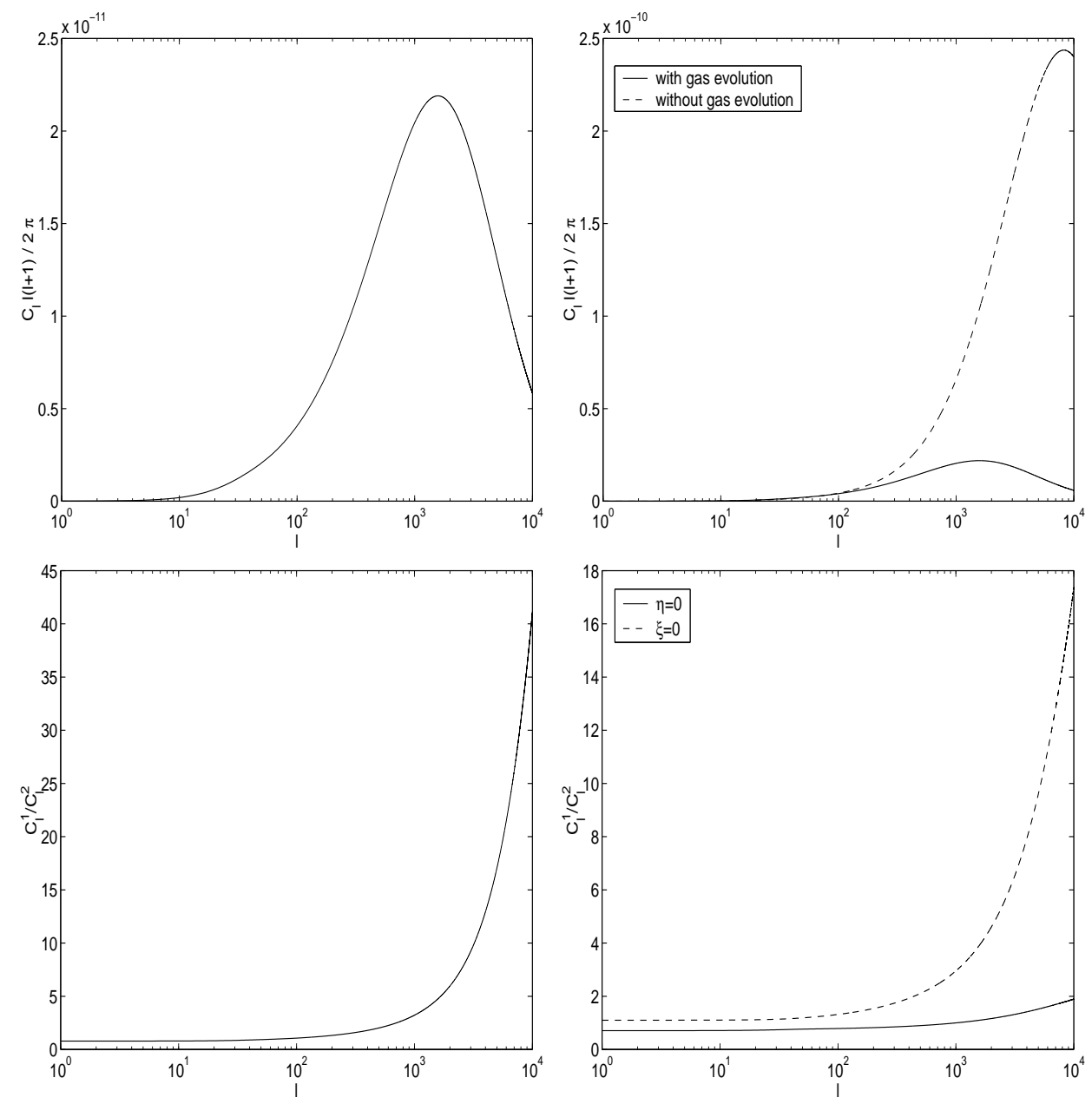

Fig. 1. The SZ angular power spectrum in the $\Lambda \mathrm{CDM}$ model, with gas evolution (upper left panel) and compared to a non-evolving gas scenario (upper right panel). The lower left panel depicts the ratio between the two power spectra; the lower right panel describes the ratio between the power spectrum obtained in the non-evolving IC gas scenario and two 'partial' IC gas evolving models. 


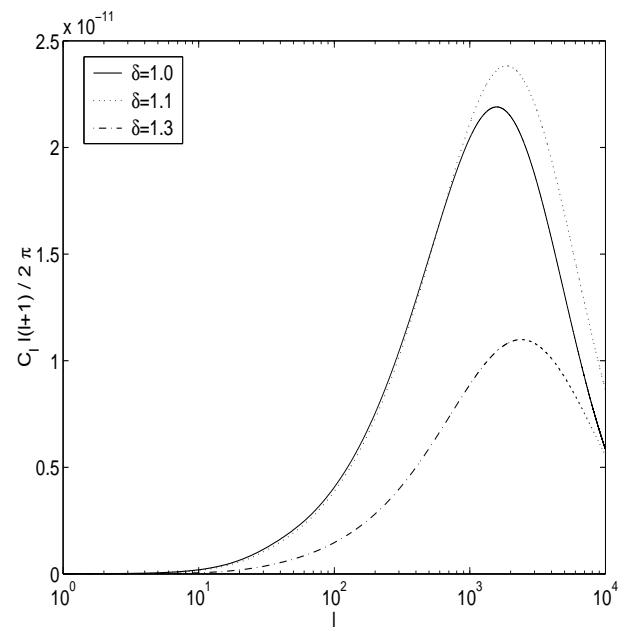

Fig. 2. SZ angular power spectrum for isothermal $(\delta=1.0)$ gas and two polytropic temperature profiles with $\delta=1.1$ and 1.3.
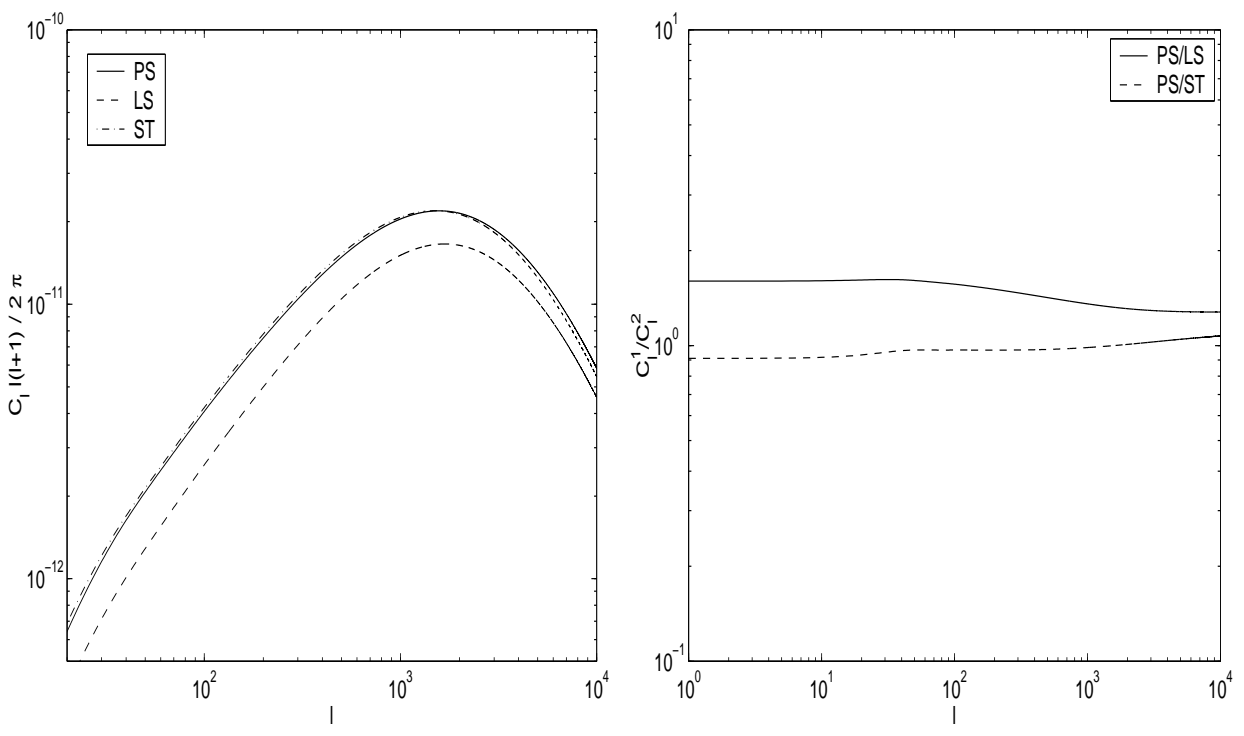

Fig. 3. SZ power spectrum calculated using the PS, LS and ST mass functions 


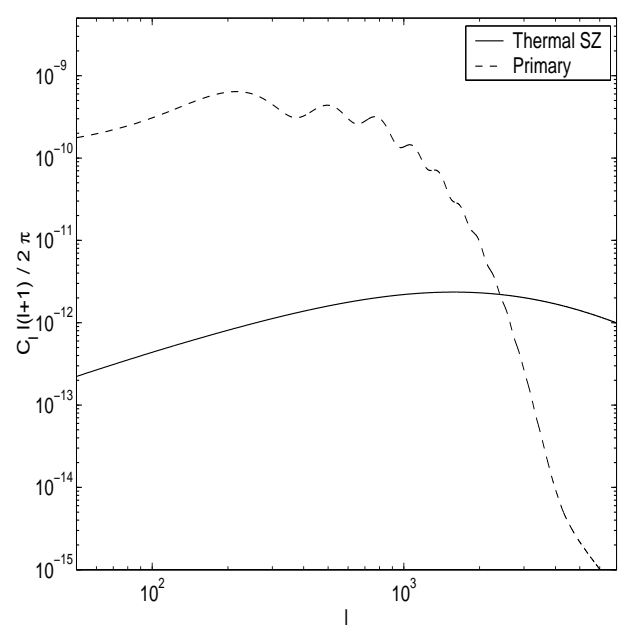

Fig. 4. SZ and primary anisotropy power spectra in the $\Lambda$ CDM models

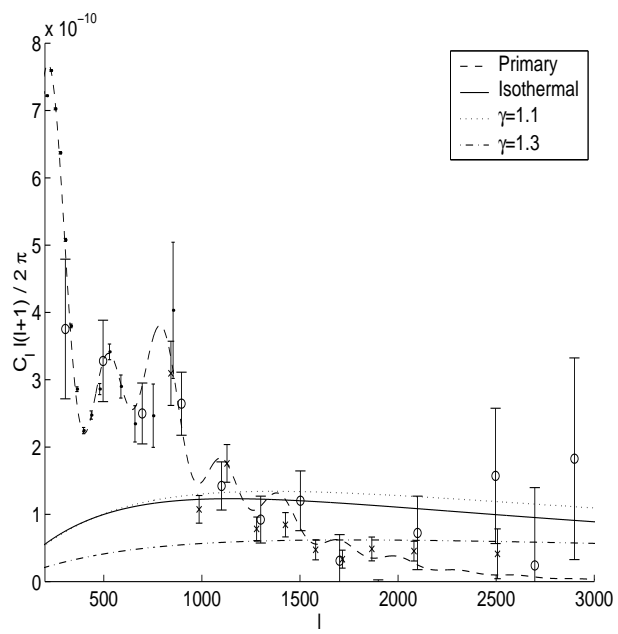

Fig. 5. Recent results from the CBI, ACBAR, and WMAP experiments, together with the angular power spectrum of the primary anisotropy as calculated by CMBFAST, and the SZ power spectrum in isothermal and polytropic models. WMAP, ACBAR, and CBI data are marked by dots, crosses, and empty circles, respectively. 

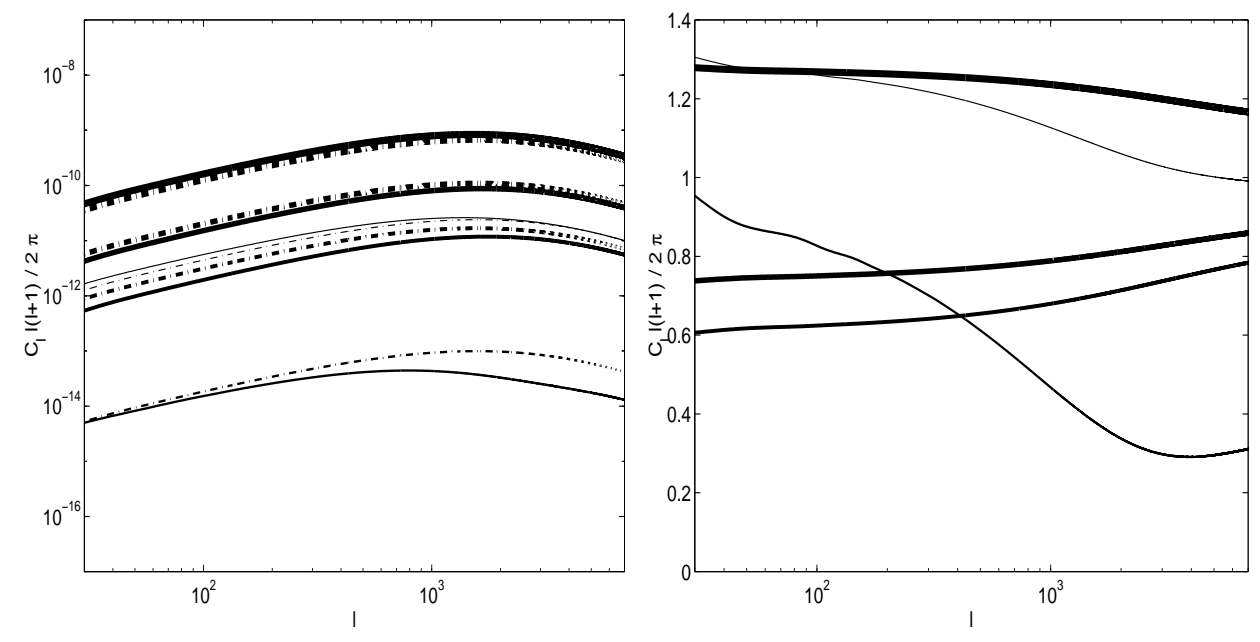

Fig. 6. Power spectra calculated using the non-relativistic and exact relativistic SZ intensity change are shown in the left panel. The spectral dependence was calculated at five frequencies, $x=2.5,3.9,4.8,6.2,9.6$; each pair of adjacent solid and dash-dotted lines correspond to the relativistic and non-relativistic calculation, respectively. Line thickness increases with the value of $x$. Ratios of relativistic to non-relativistic power at each frequency are shown in the right panel.
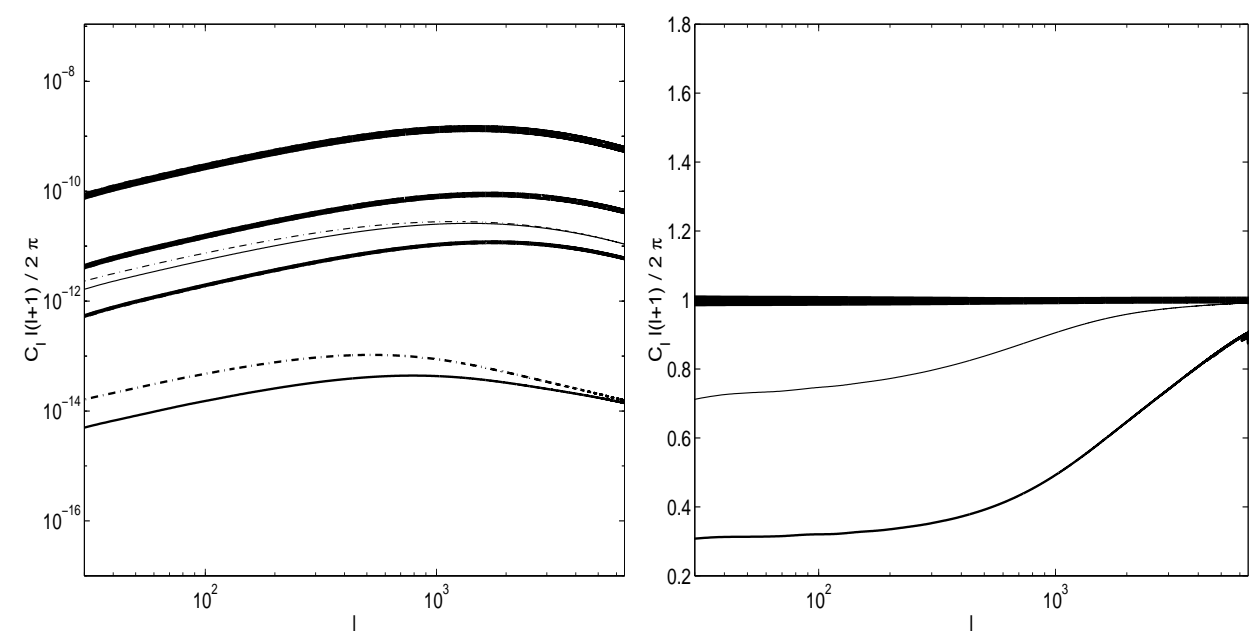

Fig. 7. Comparison of the results from the exact relativistic and approximate analytic expressions. Solid lines show the power spectrum calculated using the exact relativistic calculation of Rephaeli (1995b) for the spectral function, whereas the dash-dotted lines show the corresponding results when the analytic expression of Itoh et al (1998) is used. Ratios of the results from the exact relativistic calculation to those from the analytic approximation are shown in the right panel; line thickness corresponds to frequency as in the previous figure. 


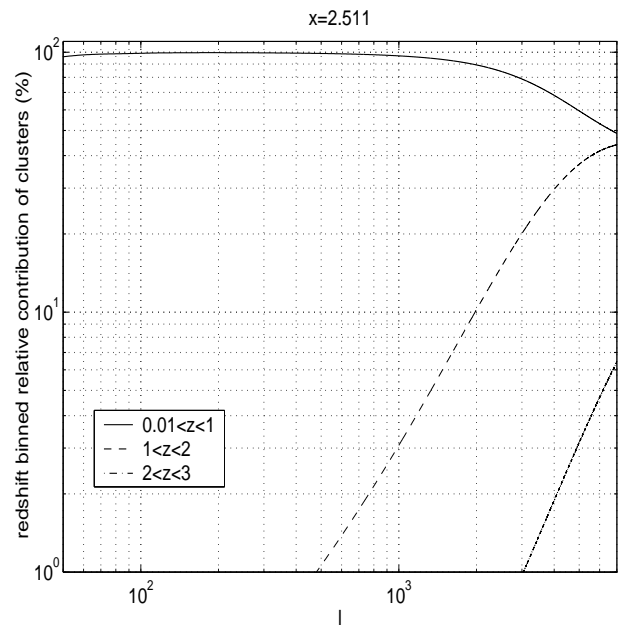

Fig. 8. Relative contribution to the overall power spectrum, at $x=2.5$, by clusters at three redshift intervals. 